\title{
Distribution of Intrarenal Blood Flow following Aortic Clamping and Declamping
}

\author{
Jack L. Cronenwett, M.D., and S. Martin Lindenauer, M.D. \\ Department of Surgery, University of Michigan, and Vascular Research Laboratory, Veterans \\ Administration Hospital, Ann Arbor, Michigan 48105
}

Submitted for publication November 9, 1976

Acute renal failure has frequently been reported following operations which require cross-clamping the abdominal aorta below the renal arteries. In the past 10 years, incidences of up to $47 \%$ decreased renal function, $37 \%$ oliguria, and $11 \%$ mortality have been reported [30]. Although modern developments in patient care, anesthesia, and operative technique have reduced the incidence of renal failure to $3-5 \%[20,40]$, the mortality of this complication approaches $25 \%$ despite aggressive therapy [1]. Recent measures to prevent this sequela have focused on adequate replacement of crystalloid during operation. Using this technique, Thompson et al. reported a mortality from renal failure of only $0.2 \%$ in 673 cases of aortoiliac surgery [41].

Despite these improvements, the underlying etiology of renal failure following aortic surgery remains obscure. Early animal experiments demonstrated acute tubular necrosis, which was attributed to aortic crossclamping, per se, and was reversed by direct or pharmacologic renal nerve blockade [27,31]. Subsequent investigators, however, were unable to substantiate these findings [5, 17, 20, 22], and it was generally concluded that aortic cross-clamping has no detrimental renal effect separate from laparotomy alone $[9,15]$. Recently, however, reports by Abbott and Berkowitz and their colleagues have contested this conclusion [1, 2]. Using the ${ }^{133} \mathrm{Xe}$ washout technique to measure the distribution of intrarenal blood flow, these investigators demonstrated decreased cortical blood flow with increased juxtamedullary and outer medullary flow during aortic crossclamping. They suggested that this technique detects more subtle renal damage than previously appreciated by other measurements and that, in fact, aortic crossclamping does contribute to renal complications.

Recently, microsphere techniques have been introduced to quantitate the distribution of intrarenal blood flow directly. Several investigators have compared the microsphere and xenon washout techniques and have reported discrepancies, especially under abnormal physiologic conditions [23, $35,37,39]$. Criticism of the xenon technique prompted the present study, which was designed to analyze the distribution of intrarenal blood flow during aortic clamping and declamping using a direct microsphere technique. The results of this study do not support previously reported shifts of intrarenal blood flow measured by xenon-133 washout.

\section{METHODS}

Six female dogs ( 16 to $25 \mathrm{~kg}$ ) were anesthetized with intravenous pentobarbital sodium $(30 \mathrm{mg} / \mathrm{kg})$. The dogs were ventilated with a Harvard respirator and supplied with low flow oxygen. Initial esophageal temperature was maintained with intermittent heat from a thermal mattress and a heat lamp, continuously regulated by a Yellow Springs Model 71 controller. Mean arterial pressure was monitored via a right brachial 
artery catheter. A Swan-Ganz thermal dilution catheter was inserted into the pulmonary artery via the external jugular vein. Cardiac output was then measured using an Edwards Model 9500 computer. The left brachial artery was cannulated with 0.055 in.-i.d. polyethylene tubing to provide one site for withdrawal of microsphere reference samples. Laparotomy was performed through a midline incision. Both ureters wcre catheterized to collect individual kidney urine samples. The left renal artery was dissected minimally to allow placement of a 3- to 4-mm electromagnetic flow probe, with care taken to preserve the renal nerves. Using a Statham SP 2201 flowmeter, left renal artery flow was continuously monitored. Electrical zero was obtained repeatedly and probes were calibrated on ap- propriate-sized vessels in vivo. Both renal veins were cannulated with 18.5 -gauge polyethylene tubing directed with a guide wire after insertion through the vena cava. A superior mesenteric artery branch was cannulated with 0.055 -in.-i.d. polyethylene tubing, with the tip of the catheter positioned just into the aorta, to provide the second catheter for withdrawal of microsphere reference samples. A splenic artery branch was used to insert a 7F side-hole cardiac catheter which was positioned in the left ventricle by pressure recording. The aorta was dissected for a short distance immediately below the renal arteries and a clamp was placed loosely around it for later occlusion at a level $1 \mathrm{~cm}$ below the left renal artery. This arrangement of catheters, as shown in Fig. 1, allowed micro-

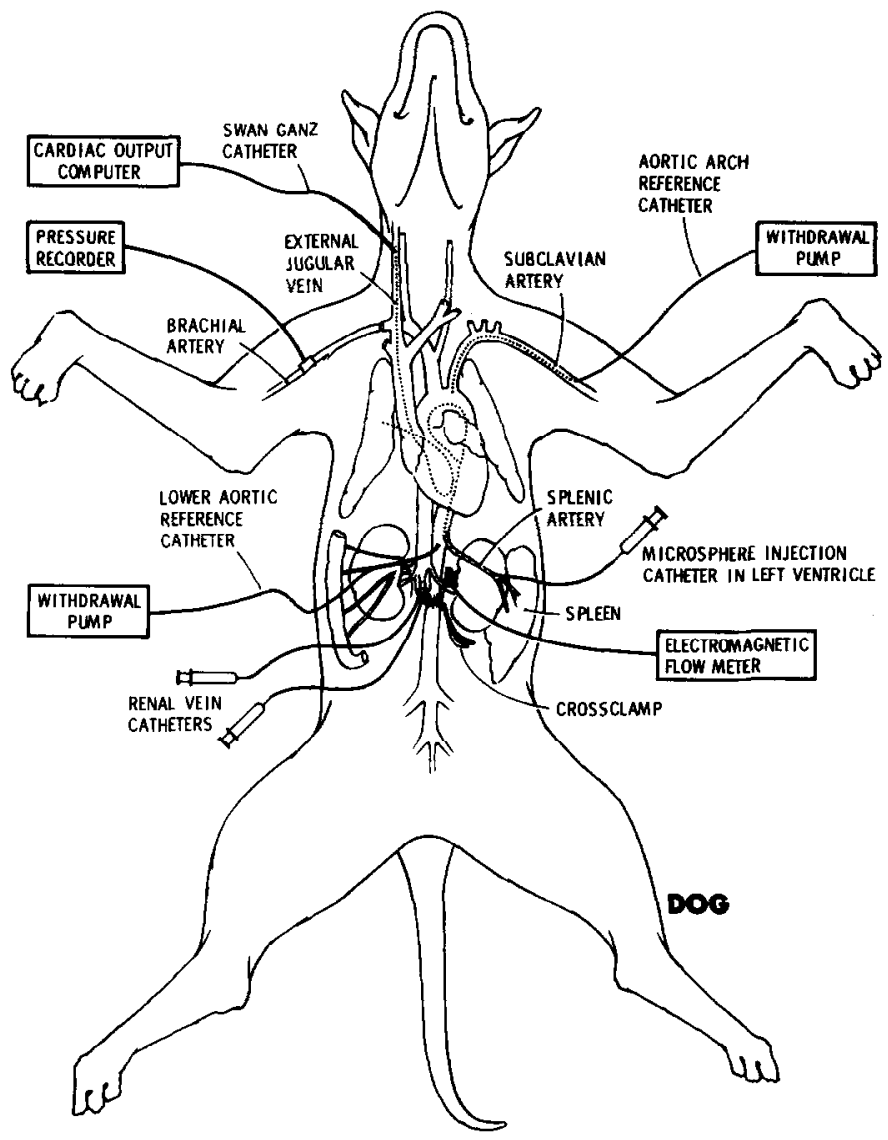

FIG. 1. Animal preparation showing catheter placement to allow aortic cross-clamping while not disturbing carotid arteries. 
sphere injection and reference sampling from both the upper and lower aorta despite aortic cross-clamping. Furthermore, in this preparation the carotid arteries were not disturbed, thus eliminating any possible effect of carotid body manipulation on renal blood flow distribution. Data were recorded continuously on a Hewlett-Packard Series 8800 recorder.

To insure accurate determination of creatinine clearance, dogs received an initial dose and then a constant infusion of creatinine, calculated to maintain a serum level of $5 \mathrm{mg} \%$. During laparotomy, animals received $500 \mathrm{ml}$ of Ringers' lactate solution. During the entire experiment, Ringers' lactate was infused at $2 \mathrm{ml} / \mathrm{min}$ to establish adequate urine output. Animals were heparinized with 100 units/kg. Following laparotomy, $1 \mathrm{hr}$ was allowed for equilibration. The aorta was then clamped, as described above, for $90 \mathrm{~min}$. After declamping, data were collected for $60 \mathrm{~min}$. Urine samples were obtained in 15 -min sampling intervals, illustrated in Fig. 2. Hemodynamic data were recorded continuously, but appropriate points were chosen for sequential analysis as seen in Fig. 2, with each point representing the 2 -min average at that time. Renal venous samples for renin assay were drawn $5 \mathrm{~min}$ before clamping, $5 \mathrm{~min}$ before declamping, and $55 \mathrm{~min}$ after declamping. Care was taken to avoid blood withdrawal $10 \mathrm{~min}$ prior to renin sampling. Renin activity was determined by radioimmunoassay (angiotensin 1, Clinical Assays, Inc.). Serum samples were drawn at appropriate intervals for sodium and creatinine determination. All blood drawn was replaced with an equal volume of saline.

Radioactive microspheres were used to measure the intrarenal distribution of blood flow as well as total renal blood flow to each kidney, immediately before clamping, immediately before declamping, and $1 \mathrm{~h}$ after declamping, using a different isotope for each determination. Carbonized microspheres, labeled with ${ }^{51} \mathrm{Cr},{ }^{141} \mathrm{Ce}$, and ${ }^{85} \mathrm{Sr}$, with a diameter of $15 \pm 5 \mu \mathrm{m}$, and suspended in $20 \%$ dextran, were used (Nuclear Products, 3M Co.). At the time of flow determination, 400,000 microspheres were injected into the left ventricular catheter and flushed through the catheter with $20 \mathrm{ml}$ of heparinized saline over $10 \mathrm{sec}$. Reference samples of blood were withdrawn from the upper and lower aortic catheters simultaneously using a Harvard withdrawal pump at the rate of $20 \mathrm{ml} / \mathrm{min}$, for $1 \mathrm{~min}$, beginning $5 \mathrm{sec}$ before microsphere injection. Using the reference-sampling technique [3], renal blood flow was then calculated for each kidney as described below, and the activities of microspheres in the upper and lower aortic sample were compared as an index of proper mixing and homogenous distribution. No change in blood pressure or renal artery flow occurred during this microsphere injection and reference-sampling period.

At the conclusion of the declamp period, the dogs were sacrificed, and the kidneys were removed and weighed. The entire kidney and the aortic reference samples were placed into plastic containers to provide equivalent geometry and they were counted in a Nuclear Chicago single-sample well detector and pulse height analyzer.

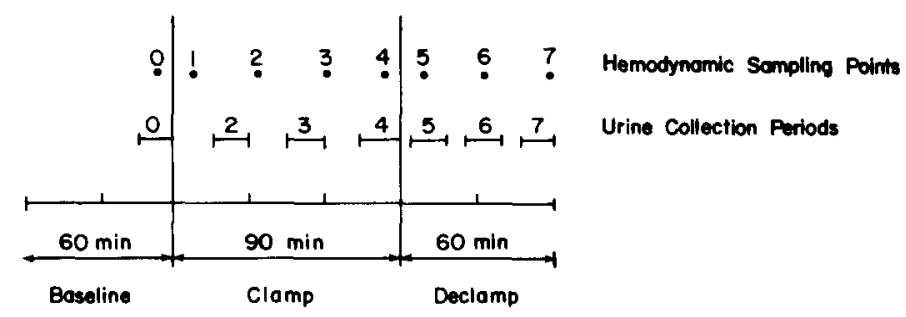

FIG. 2. Urine sampling periods ( $15 \mathrm{~min})$ and hemodynamic analysis points (2-min mean at each point). 
Isotope separation in kidney samples was accomplished by solving multiple simultaneous linear equations as described by $\mathrm{Ru}-$ dolph and Heymann [34]. Total renal blood flow (RBF) to each kidney was then calculated by the formula:

$$
\mathrm{RBF}=\frac{\mathrm{cpm}_{\mathrm{k}}}{\mathrm{cpm}_{\mathrm{r}}} \times V_{\mathrm{r}}(\mathrm{ml} / \mathrm{min}),
$$

where $\mathrm{cpm}_{\mathrm{k}}=$ isotope counts per minute, total kidney; $\mathrm{cpm}_{\mathrm{r}}=$ isotope counts per minute, aortic reference sample; $V_{\mathrm{r}}=$ volume of reference sample withdrawn in 1 $\min$.

Following isotope counting of the entire kidney, each kidney was divided in half coronally. Thin sections were taken in coronal fashion from three different areas of cortex of each half-kidney. These blocks of cortex (approximately 4-mm thick $\times 10$ $\mathrm{mm}$ wide) were then cut into four equal layers (from outer to inner cortex) labeled as Layers 1 to 4 . It was found that equal layers could be cut by hand from fresh tissue after familiarity was obtained with this technique. Three samples of each layer from each half-kidney were pooled to decrease sectioning and anatomic variation. Samples from each half of each kidney were kept separate to provide an analysis of variance of this technique in each animal. Samples were placed in preweighed airtight vials and subsequently weighed prior to counting. The remaining kidney was then fixed for histologic examination. The sample vials of each pooled cortical layer from each half-kidney were then counted for each isotope simultaneously using a lithium drifted germanium detector, capable of the high resolution necessary to separate the three isotopes. To remove any geometric distortion, all samples were rotated during the 1000 -sec counting time. On-line computer analysis resolved each isotope activity and subtracted background, to provide overall count rates with $<4 \%$ error.

The uncorrected percentage distribution of renal blood flow to each cortical layer was calculated as the percentage of isotope (microspheres) distributed to each of the four layers, normalizing each layer for the weight of the counted tissue. Thus,

$\%$ distribution to Layer $i$

$$
=\frac{\left(C_{i} / W_{i}\right)}{\sum_{i=1}^{4}\left(C_{i} / W_{i}\right)} \times 100,
$$

where $C_{i}=$ isotope counts per unit time for Layer $i$, and $W_{i}=$ actual tissue weight of Layer $i$.

By representing the kidney as an ellipsoid it can be calculated that the outer cortical layers occupy more total mass of the kidney than the inner layers, because all layers are of equal thickness but are distributed at different distances from the center of the ellipsoid. McNay and Abe determined the relative total kidney volumes of the four cortical zones, zone 1 to zone 4 to be $27,22,17$, and $12 \%$, respectively [23]. Total renal blood flow was equated with cortical blood flow, an assumption previously validated $[19,23,38]$. Then, the corrected distribution of blood flow within the total kidney cortex was calculated by this formula:

Corrected $\%$ distribution to Zone $i$

$$
=\frac{\left(C_{i} / W_{i}\right) \times Z_{i}}{\sum_{i=1}^{4}\left[\left(C_{i} / W_{i}\right) \times Z_{i}\right]} \times 100,
$$

where $C_{i}=$ isotope counts per unit time for Layer $i, W_{i}=$ tissue weight Layer $i$, and $Z_{i}=$ fraction of kidney occupied by Zone $i$ (above).

Zonal perfusion rate normalized for weight and relative zone mass was calculated by the equation:

Perfusion rate $=\frac{\mathrm{RBF} \times F_{i}}{W_{k} \times Z_{i}} \quad(\mathrm{ml} / \mathrm{min} / \mathrm{g})$,

Where $\mathrm{RBF}=$ renal blood flow, $F_{i}=$ corrected percentage distribution to Zone $i$ expressed as a fraction, $W_{k}=$ weight of kidney, and $Z_{i}=$ fraction of kidney occupied by Zone $i$. 
TABLE 1

Hemodynamic Data ${ }^{a}$

\begin{tabular}{|c|c|c|c|c|c|c|c|c|}
\hline & \multirow{2}{*}{$\begin{array}{l}\text { Baseline } \\
\qquad 0^{b}\end{array}$} & \multicolumn{4}{|c|}{ Cross-clamp } & \multicolumn{3}{|c|}{ Declamp } \\
\hline & & 1 & 2 & 3 & 4 & 5 & 6 & 7 \\
\hline $\begin{array}{l}\text { Cardiac index } \\
\text { (liters } / \mathrm{min} / \mathrm{m}^{2} \text { ) }\end{array}$ & $3.1 \pm 0.2$ & $2.9 \pm 0.2$ & $2.9 \pm 0.3$ & $2.8 \pm 0.3$ & $2.9 \pm 0.3$ & $3.1 \pm 0.3$ & $3.0 \pm 0.2$ & $3.1 \pm 0.2$ \\
\hline $\begin{array}{l}\text { Mean arterial } \\
\text { pressure (mm } \mathrm{Hg})\end{array}$ & $149 \pm 3$ & $151 \pm 3$ & $155 \pm 4$ & $156 \pm 5$ & $152 \pm 4$ & $148 \pm 5$ & $145 \pm 4$ & $143 \pm 4$ \\
\hline $\begin{array}{l}\text { Left renal artery } \\
\text { blood flow (ml/ } \\
\text { min) }\end{array}$ & $236 \pm 24$ & $231 \pm 25$ & $221 \pm 27$ & $217 \pm 28$ & $223 \pm 25$ & $232 \pm 25$ & $235 \pm 21$ & $239 \pm 22$ \\
\hline
\end{tabular}

a Values are means \pm SEM. No values are statistically different from baseline.

${ }^{b}$ The numbers $0-7$ are sampling periods.

All data were subjected to statistical analysis, with the specific tests noted under Results.

\section{RESULTS}

Reference samples from the upper and lower aorta were compared for differences in isotope content. The mean difference in activity was $11 \%( \pm 2 \%$, SEM). For renal blood flow calculations, the lower aortic sample was selected for its proximity to the renal artery. When the two halves of each kidney within all dogs were analyzed, the maximum difference of isotope activity between kidney halves was $15 \%$.

Cardiac index, mean arterial pressure, and left renal artery blood flow (electromagnetic flow meter) did not change significantly between baseline and clamp or declamp period, as shown in Table 1 and Fig. 3. There was no difference in any of these parameters in any time period, with the exception that blood pressure in Periods 3 and 4 was slightly higher than in Period $7(P<0.05) .{ }^{1}$ However, these three periods were not significantly different from baseline values. It should be noted that in each animal there was a transient increase in arterial pressure (mean, $5 \mathrm{~mm} \mathrm{Hg}$; duration, $1 \mathrm{~min}$ ) immediately after clamping.

\footnotetext{
1 Two-way analysis of variance with mixed effects. Tukey pairwise comparison for individual significant contrasts.
}

Furthermore, immediately after declamping there was a transient decrease in blood pressure (mean, $8 \mathrm{~mm} \mathrm{Hg}$; duration 1.5 , min). Because of the sampling periods chosen for analysis, these transient variations are not shown in Fig. 3.

Renal function parameters were quite constant over time as shown in Table 2 and Fig. 4. Right and left kidneys were analyzed separately, but none of the parameters during any time period was significantly ${ }^{2}$ different between kidneys. Therefore, data from these kidneys were averaged to provide a mean individual kidney value, as depicted in Table 2 . Urine output was unchanged during clamp

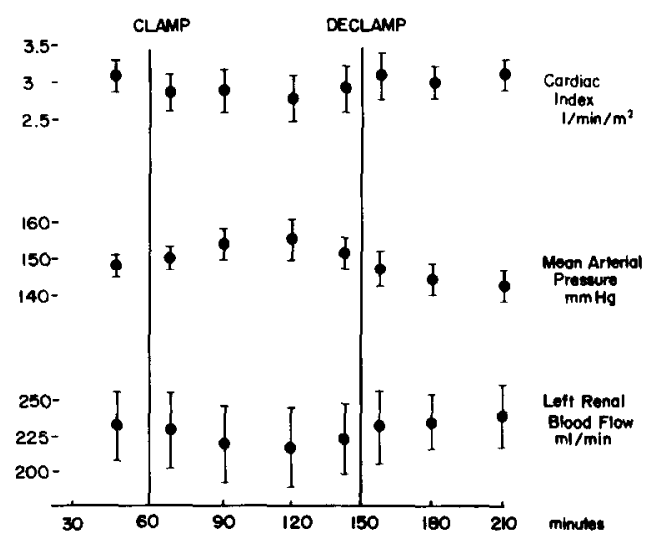

FIG. 3. Hemodynamic data during baseline, crossclamp, and declamp periods.

${ }^{2}$ Multivariant $\boldsymbol{F}$ test between kidneys. One-way multivariant analysis of variance over time. 
TABLE 2

Renal Function Data ${ }^{a}$

\begin{tabular}{|c|c|c|c|c|c|c|c|}
\hline & \multirow{2}{*}{$\begin{array}{c}\text { Baseline } \\
0^{b}\end{array}$} & \multicolumn{3}{|c|}{ Cross-clamp } & \multicolumn{3}{|c|}{ Declamp } \\
\hline & & 1 & 3 & 4 & 5 & 6 & 7 \\
\hline $\begin{array}{l}\text { Urine output } \\
(\mathrm{ml} / \mathrm{min})\end{array}$ & $1.4 \pm 0.2$ & $0.9 \pm 0.2$ & $0.8 \pm 0.2$ & $0.8 \pm 0.2$ & $0.8 \pm 0.2$ & $0.9 \pm 0.2$ & $1.0 \pm 0.2$ \\
\hline $\begin{array}{l}\text { Creatinine clear- } \\
\text { ance }(\mathrm{ml} / \mathrm{min})\end{array}$ & $50 \pm 3$ & $49 \pm 4$ & $45 \pm 3$ & $47 \pm 3$ & $52 \pm 4$ & $52 \pm 4$ & $53 \pm 3$ \\
\hline $\begin{array}{l}\text { Sodium excretion } \\
\text { (mequiv/min) }\end{array}$ & $133 \pm 34$ & $115 \pm 24$ & $90 \pm 18$ & $82 \pm 20$ & $92 \pm 15$ & $90 \pm 18$ & $91 \pm 18$ \\
\hline
\end{tabular}

${ }^{a}$ Values are means \pm SEM for single kidney derived as mean right and left kidney; urine output: baseline $>$ cross-clamp $=$ declamp $(P<0.05)$; no other significant changes.

${ }^{b}$ The numbers $0-7$ are sampling periods.

and declamp periods, but was slightly higher during Period 0 (bascline) ( $P$ $<0.05) .{ }^{1}$ Creatinine clearance was unchanged from baseline during clamp and declamp periods. ${ }^{1}$ Sodium excretion was not significantly different in any time interval. ${ }^{1}$ Serum creatinine and serum sodium were constant throughout the procedure.

Renal blood flow in both kidneys determined by microsphere reference sampling was compared with left renal artery flow measured by the electromagnetic flowmeter, as shown in Table 3 and Fig. 5. There was no significant difference in blood flow between right and left kidneys (microsphere technique) or blood flow to the left

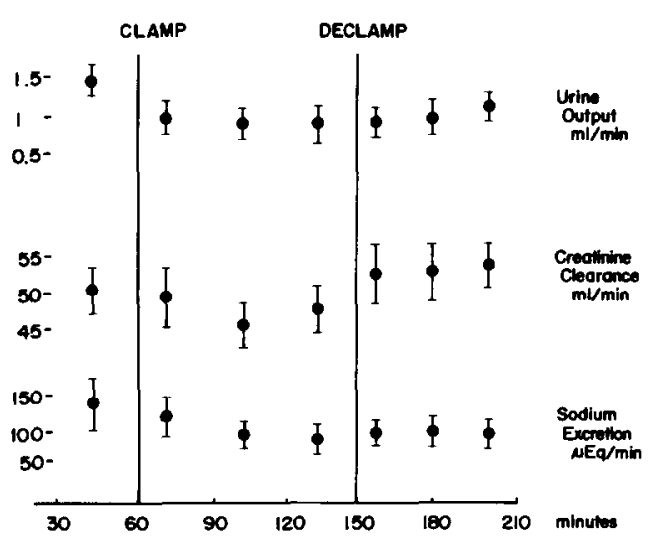

FIG. 4. Renal function data during baseline, crossclamp and declamp periods (means, right and left kidney). kidney determined by microsphere versus flowmeter tcchniquc. ${ }^{3}$ Right and left renal blood flows were highly correlated with $r=0.88, P<0.001$ (linear regression).

Plasma renin activity did not change significantly between baseline, clamp, or declamp period in either kidney or in the mean value of both kidneys, as shown in Table 4. ${ }^{4}$ Furthermore, there was no difference between the right and left kidney at any sampling time.

Intrarenal distribution of blood flow expressed as uncorrected percentage is shown in Table 5 and Fig. 6. During all time intervals, there was a significant difference in percent distribution between Layers 1, 2, 3, and 4, with greatest distribution (36\%) to outer cortex (Layer 1), decreasing successively to the least distribution (15\%) to inner cortex (Layer 4) with $P$ $<0.05 .^{4}$ Distribution of blood flow was compared in each layer between kidneys, but no significant difference could be determined. Therefore, the mean value for both kidneys is represented in Fig. 5. The distribution of blood flow to these layers did not change significantly from baseline to clamp to declamp time. ${ }^{5}$ The same was true

${ }^{3}$ Interclass correlation coefficient.

${ }^{4}$ Two-way analyses of variance without replication.

${ }^{5}$ Friedman rank sum with multiple comparison. Multiple comparison procedure to separate significant individual contrasts. 


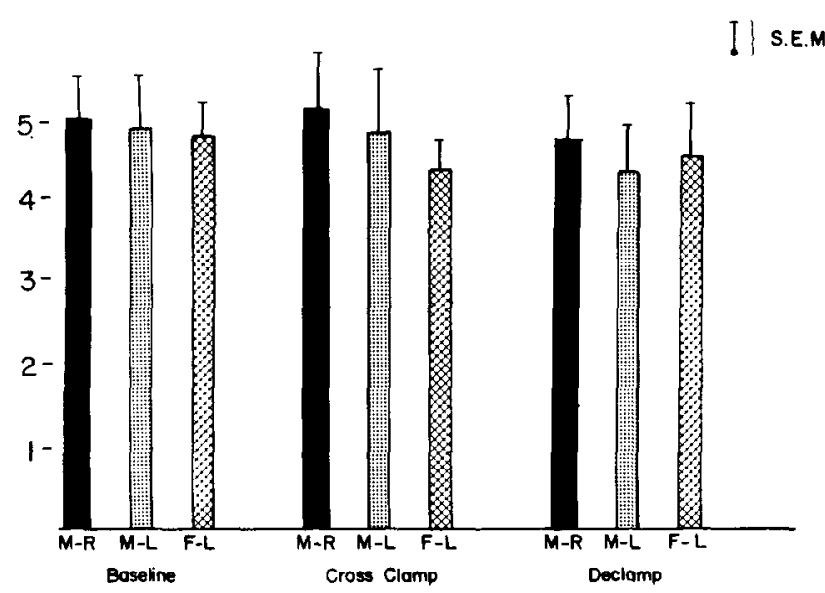

FIG. 5. Renal blood flow (milliliters per minute per gram) during baseline, cross-clamp, and declamp periods at times noted in text. M-R: right kidney, microsphere technique; M-L: left kidney, microsphere technique; F-L: left kidney, electromagnetic flow meter.

of each kidney analyzed individually with the only exception being Layer 2 of the right kidney where there appeared to be slightly increased distribution at declamp time (30.5\%) from baseline $(27.5 \%), P<0.05 .^{5}$ Distribution during clamp time, however, was not different from baseline or declamp time in this layer.

The corrected percentage distribution of blood flow to each total zone was treated identically to uncorrected distribution and is shown in Table 6 and Fig. 7. The results were identical to uncorrected distribution; that is, a difference existed between distribution to Zones 1, 2, 3, and 4 at all three intervals but no difference existed in zonal distribution between baseline, clamp, and declamp intervals (with the above exception

TABLE 3

ReNal BloOd Flow ${ }^{a}$

\begin{tabular}{|c|c|c|c|}
\hline \multirow[b]{2}{*}{ Flow } & \multicolumn{2}{|c|}{ Microsphere reference } & \multirow{2}{*}{$\begin{array}{c}\text { Flow probe } \\
\text { left }\end{array}$} \\
\hline & Right & Left & \\
\hline \multicolumn{4}{|c|}{ Total flow ( $\mathrm{ml} / \mathrm{min})$} \\
\hline Baseline & $235 \pm 33$ & $253 \pm 51$ & $241 \pm 23$ \\
\hline Cross-clamp & $236 \pm 25$ & $231 \pm 29$ & $217 \pm 25$ \\
\hline Declamp & $225 \pm 22$ & $215 \pm 25$ & $241 \pm 23$ \\
\hline \multicolumn{4}{|c|}{ Flow per gram $(\mathrm{ml} / \mathrm{min} / \mathrm{g})$} \\
\hline Baseline & $4.89 \pm 0.55$ & $4.83 \pm 0.70$ & $4.76 \pm 0.46$ \\
\hline Cross-clamp & $4.99 \pm 0.60$ & $4.74 \pm 0.73$ & $4.66 \pm 0.59$ \\
\hline Declamp & $4.71 \pm 0.34$ & $4.29 \pm 0.37$ & $4.53 \pm 0.34$ \\
\hline
\end{tabular}

${ }^{n}$ Values are means \pm SEM. No values are statistically different.
TABLE 4

Plasma Renin Activity ${ }^{a}$

\begin{tabular}{lcrr}
\hline & $\begin{array}{c}\text { Baseline } \\
(\mathrm{ng} / \mathrm{ml} / \mathrm{hr})\end{array}$ & $\begin{array}{c}\text { Cross-clamp } \\
(\mathrm{ng} / \mathrm{ml} / \mathrm{hr})\end{array}$ & $\begin{array}{c}\text { Declamp } \\
(\mathrm{ng} / \mathrm{ml} / \mathrm{hr})\end{array}$ \\
\hline $\begin{array}{l}\text { Right kidney } \\
\text { Left kidney }\end{array}$ & $5.9 \pm 1.3$ & $10.5 \pm 3.4$ & $8.1 \pm 2.0$ \\
$\begin{array}{l}\text { Mean, R \& L. } \\
\text { kidney }\end{array}$ & $5.7 \pm 1.9$ & $7.1 \pm 2.4$ & $8.3 \pm 2.3$ \\
\hline
\end{tabular}

$a$ Values are means \pm SEM. No values statistically different from baseline.

noted to Zone 2, right kidney, declamp period)..$^{5}$

Intrarenal tissue perfusion, or the actual rate of blood flow to each cortical zone, is shown in Table 7 and Fig. 8. Again there was a significant difference in the flow rate to Zones 1, 2, 3, and 4, with greatest flow to outer cortex and least flow to inner cortex, with $P<0.05 .^{5}$ Furthermore, there was no change in flow to any Zone during baseline, clamp, or declamp periods. ${ }^{5}$ The minor change in distribution to Zone 2 of the right kidney was not significant when the distribution was corrected to actual tissue perfusion (milliliters per minute per gram).

Histological examination of each kidney showed no evidence of renal cortical or tubular damage. Microspheres distributed 
TABLE 5

Distribution of INTRARENAL BLOOD Flow ${ }^{a}$

\begin{tabular}{ccccc}
\hline & & \multicolumn{3}{c}{ Uncorrected (\%) } \\
\cline { 3 - 5 } & Layer & Baseline & Cross-clamp & Declamp \\
\hline Right kidney & 1 & $37.5+2.0$ & $40.0+3.1$ & $37.2+2.4$ \\
& 2 & $27.5 \pm 0.8^{*}$ & $28.8 \pm 0.8$ & $30.5 \pm 0.8^{*}$ \\
& 3 & $20.8 \pm 1.0$ & $19.0 \pm 1.3$ & $19.5 \pm 1.4$ \\
& 4 & $15.0 \pm 1.5$ & $13.7 \pm 2.0$ & $13.2 \pm 1.4$ \\
Left kidney & 1 & $34.0 \pm 2.7$ & $34.3 \pm 3.1$ & $32.3 \pm 2.7$ \\
& 2 & $29.0 \pm 1.0$ & $28.7 \pm 1.0$ & $29.2 \pm 0.9$ \\
& 3 & $23.2 \pm 2.3$ & $23.3 \pm 2.2$ & $24.5 \pm 2.4$ \\
Mean, R and & 4 & $15.2 \pm 0.7$ & $15.0 \pm 1.5$ & $15.2 \pm 1.7$ \\
L kidney & 1 & $35.8 \pm 2.4$ & $37.2 \pm 3.2$ & $34.8 \pm 2.7$ \\
& 3 & $28.3 \pm 0.9$ & $28.8 \pm 0.9$ & $29.8 \pm 0.9$ \\
& 4 & $22.0 \pm 1.8$ & $21.2 \pm 2.0$ & $22.0 \pm 2.2$ \\
& $15.1 \pm 1.1$ & $14.3 \pm 1.7$ & $14.2 \pm 1.6$ \\
\hline
\end{tabular}

${ }^{a}$ The values are means \pm SEM.

* Only significantly different value $(P<0.05)$ within each layer.

only to glomeruli or afferent arterioles, with rarely more than one and never more than two spheres together in one arteriole. Since only $5-10 \%$ of glomeruli contained microspheres after all injections, glomerular perfusion should not have been significantly affected by microsphere obstruction.

\section{DISCUSSION}

This study has demonstrated that cardiac index, arterial pressure, and renal blood flow are constant despite intrarenal aortic clamping and declamping in normal dogs. Creatinine clearance and sodium excretion were both normal and stable. After an initial increase in urine volume following a saline bolus, urine output was constant during aortic clamping and declamping. It should be noted that the extracellular space was supported in this study by a constant infusion of Ringers' lactate at $2 \mathrm{ml} /$ min and by an initial 500-ml Ringers' lactate bolus to insure adequate replacement of operative fluid and blood losses. Previous reports of decreased renal blood flow, creatinine clearance, and urine output generally occurred when there was no saline or when insufficient saline was continuously administered over the course of the experiment $[5,15,17]$. When continuous saline infusions were provided, however, no change in these parameters was noted, as was true in the present study $[26,36]$. In a recent report by Stein et al. [40], a gradual decrease in renal blood flow despite saline replacement was noted during aortic clamping and declamping. This was prevented with ethacrynic acid. However, no operated control animals without clamping were studied. Several investigators have demonstrated that although a slight decrease in renal blood flow may occur with aortic cross-clamping, it does not exceed operated controls $[9,15]$. Therefore, the value of ethacrynic acid was not proven by Stein et al., and the need for this treatment was not evident in the present study. This conclusion is supported by the clinical report of Thompson et al. that adequate

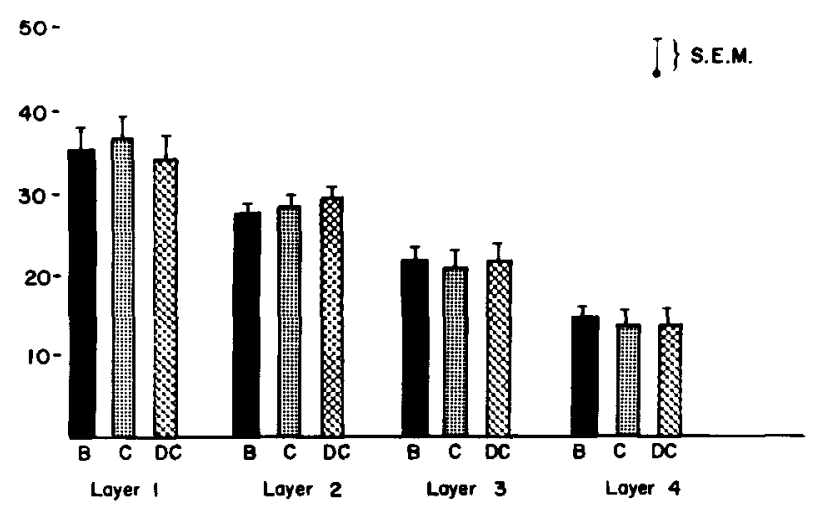

FIG. 6. Distribution of intrarenal blood flow; uncorrected percentages (means, right and left kidney). B: baseline period; C; Cross-clamp period; DC: declamp period. 
operative saline replacement without diuretic therapy greatly decreased renal complications following aortic surgery [41].

Significant declamp hypotension did not occur in this study. It has previously been demonstrated that adequate fluid therapy would avoid this sequela [28]. This supports the concept that declamp hypotension results from reactive hyperemia and vascular sequestration of blood in the legs [16].

Light microscopy in this study revealed no renal damage. Only two early reports in the literature have demonstrated acute tubular necrosis associated with aortic clamp-declamping [27, 31]. Many subsequent studies have failed to confirm these findings $[6,17,22]$. It is generally accepted that aortic cross-clamping without renal ischemia, embolization, or renal vein obstruction is not associated with significant renal pathological changes [20].

The determination of renal blood flow by the microsphere reference sample technique provides a noninvasive method which correlates highly with flow measured by an electromagnetic flowmeter, but does not necessitate disturbance of the renal pedicel. It is limited by the number of microsphere injections which can be made before affecting renal function. In this study, the two kidneys were analyzed separately to insure that dissection of the left renal artery necessary to place the flow probe did not affect renal blood flow, function, or flow
TABLE 6

Distribution of INTRARENal Blood Flow ${ }^{a}$

\begin{tabular}{ccccc}
\hline & & \multicolumn{3}{c}{ Corrected $(\%)$} \\
\cline { 3 - 5 } & Zone & Baseline & Cross-clamp & Declamp \\
\hline Right kidney & 1 & $47.3 \pm 2.1$ & $49.3 \pm 2.9$ & $46.5 \pm 2.4$ \\
& 2 & $28.5 \pm 1.1^{*}$ & $29.0 \pm 1.0$ & $31.3 \pm 0.6^{*}$ \\
& 3 & $16.5 \pm 0.9$ & $15.2 \pm 1.3$ & $15.5 \pm 1.5$ \\
& 4 & $8.5 \pm 1.0$ & $7.8 \pm 1.2$ & $7.8 \pm 1.0$ \\
Left kidney & 1 & $43.3 \pm 3.0$ & $43.5 \pm 3.3$ & $41.5 \pm 2.9$ \\
& 2 & $30.2 \pm 1.3$ & $30.0 \pm 1.2$ & $30.0 \pm 0.7$ \\
& 3 & $18.8 \pm 2.0$ & $19.0 \pm 2.2$ & $20.3 \pm 2.2$ \\
& 4 & $9.0 \pm 0.5$ & $8.5 \pm 0.9$ & $9.2 \pm 1.0$ \\
Mean, R and & 1 & $45.5 \pm 2.3$ & $46.5 \pm 2.7$ & $44.2 \pm 2.3$ \\
L. kidney & 2 & $29.5 \pm 1.0$ & $29.7 \pm 0.9$ & $31.0 \pm 0.5$ \\
& 3 & $17.8 \pm 1.3$ & $17.2 \pm 0.4$ & $18.2 \pm 1.3$ \\
& 4 & $8.8 \pm 0.7$ & $8.5 \pm 0.9$ & $8.8 \pm 1.0$ \\
\hline
\end{tabular}

"The values are means + SFM

* Only significantly different value $(P<0.05)$ within each zone.

distribution. There appeared to be minor decreases in urine output, creatinine clearance, and outer cortical perfusion in the dissected (left) kidney. However, as noted, none of these differences achieved statistical significance. Nevertheless, there is the suggestion that future studies may indicate a detrimental effect of flow probe placement.

Although renal vein renin activity has been noted to increase following abdominal operations in general [8], the specific effect of aortic cross-clamping is not clear. Berkowitz and Shetty reported increased renal vein renin activity during aortic clamping which returned to normal following de-

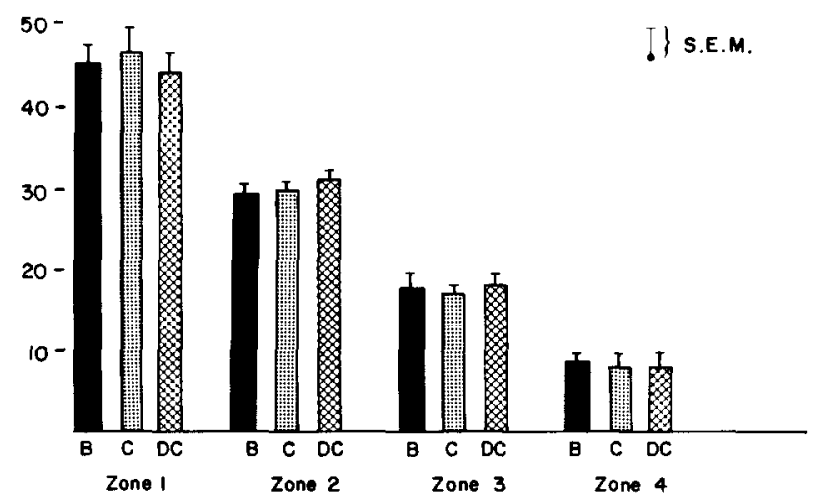

FIG. 7. Distribution of intrarenal blood flow: corrected percentages (means, right and left kidney). B: baseline period; $\mathrm{C}$ : cross-clamp period; DC: declamp period. 
TABLE 7

Intrarenal Tissue Perfusion

\begin{tabular}{ccccc}
\hline & Zone & $\begin{array}{c}\text { Baseline } \\
(\mathrm{ml} / \mathrm{min} / \mathrm{g})\end{array}$ & $\begin{array}{c}\text { Cross-clamp } \\
(\mathrm{m} / \mathrm{min} / \mathrm{g})\end{array}$ & $\begin{array}{c}\text { Declamp } \\
(\mathrm{ml} / \mathrm{min} / \mathrm{g})\end{array}$ \\
\hline Right kidney & 1 & $8.4 \pm 0.7$ & $9.2 \pm 1.4$ & $8.0 \pm 0.4$ \\
& $\mathbf{2}$ & $6.4 \pm \mathbf{0 . 9}$ & $6.6 \pm 0.7$ & $6.7 \pm 0.5$ \\
& 3 & $4.8 \pm 0.8$ & $4.3 \pm 0.5$ & $4.4 \pm 0.7$ \\
& 4 & $3.5 \pm 0.6$ & $3.2 \pm 0.6$ & $3.1 \pm 0.5$ \\
Left kidney & 1 & $7.7 \pm 1.1$ & $7.9 \pm 1.6$ & $6.7 \pm 0.8$ \\
& 2 & $6.6 \pm 1.0$ & $6.3 \pm 0.8$ & $5.8 \pm 0.4$ \\
& 3 & $5.4 \pm 1.2$ & $5.1 \pm 1.0$ & $4.9 \pm 0.6$ \\
& 4 & $3.7 \pm 0.6$ & $3.2 \pm 0.5$ & $3.3 \pm 0.5$ \\
Lean, R and & 1 & $\mathbf{8 . 1} \pm 0.9$ & $8.5 \pm 1.5$ & $7.3 \pm 0.5$ \\
& 2 & $6.5 \pm 1.0$ & $6.4 \pm 0.7$ & $6.3 \pm 0.4$ \\
& 3 & $5.1 \pm 0.9$ & $4.7 \pm 0.7$ & $4.6 \pm 0.5$ \\
& 4 & $3.6 \pm 0.6$ & $3.2 \pm 0.5$ & $3.2 \pm 0.5$ \\
\hline
\end{tabular}

${ }^{a}$ The values are means \pm SEM. No values significantly different within each zone.

clamping [7], while Stein et al. found no change in renin during these periods [40], as was the case in this report. These differences probably reffect subtle changes in the animal preparation, including state of hydration, depth of anesthesia, operative trauma, and length of experiment.

Established criteria for valid observation of the distribution of renal blood flow were met in this experiment $[11,19]$. Adequacy of left ventricular microsphere mixing was confirmed by the $11 \%$ difference in activity between upper and lower aortic reference samples, which would be expected to give the greatest divergence in cases of improper mixing. The technique of separate analyses of the layers of each half kidney estimated the range of error at a maximum of $15 \%$ in individual dogs. Microscopic examination demonstrated the proper location for microspheres within the kidney.

The distribution of intrarenal blood flow observed during the baseline period agrees well with previous observations in other laboratories by different observers $[23,24$, $33,38,39]$. It is well known that normally the greatest distribution of blood flow is to the outer cortex with gradually decreased flow to the inner cortex, where flow is the lowest. These differences are significant and agree with previous reports [23, 33, 38].

No significant change in the distribution of intrarenal blood flow during clamping or declamping was demonstrated in this study. It is probably more valid to consider corrected rather than uncorrected percentage distribution, since the former considers the entire cortical mass in zones rather than in isolated cortical segments. An equally valid measurement of blood flow distribution is the blood flow rate actually perfusing each zone. Tissue perfusion, as described in this study, provides this index. It converts relative distribution to actual flow rate, based on total renal blood flow. Using tissue perfusion as the indicator of renal cortical flow distribution, no change occurred in either kidney or in the mean value for both kidneys during baseline, clamp, or declamp determinations.

This finding of unchanged distribution

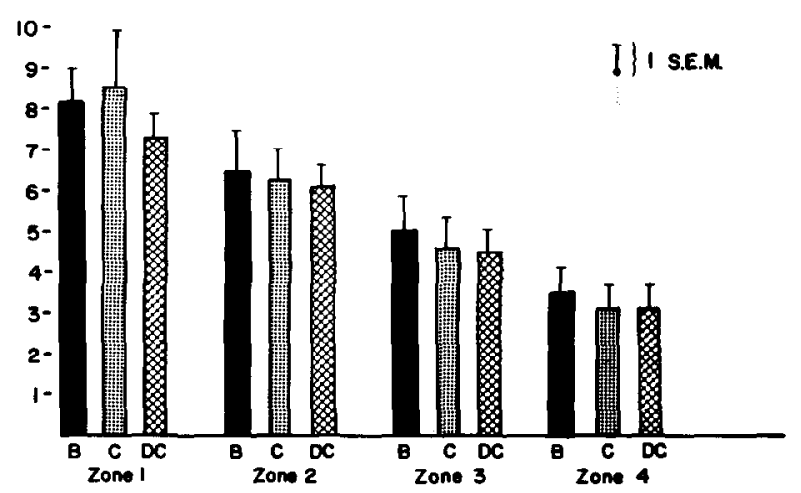

FIG. 8. Distribution of intrarenal blood flow; actual tissue perfusion to each zone (milliliters per minute per gram) (means, right and left kidney). B: baseline period; C: cross-clamp period; DC: declamp period. 
was not unexpected in light of constant hemodynamic and renal function parameters, but is at variance with previously reported findings by both Abbott and coworkers [1, 2] and Berkowitz and Shetty [7]. Several explanations for this difference exist. Abott et al. [1] infused much less saline than the present study and had very low urine output with abnormally low creatinine clearance. Furthermore, xenon-determined flow to the cortex (component $C_{\mathrm{I}}$ ) was abnormally low initially, indicating that insufficient equilibration time may have been allowed after aortic dissection. Although a reversal of the flow shift toward the juxtaglomerular cortex and medulla was reported with declamping, lack of statistical treatment does not allow independent confirmation. An alternate interpretation is that blood flow was distributed away from the cortex with increasing time in the experiment. If fluid replacement was inadequate, this is not unlike hemorrhagic hypotension in which decreased cortical flow has been demonstrated [13, 33, 37]. In the subsequent paper by Abbott and Austen [2], mannitol was infused directly into the renal artery concurrent with clamping the aorta, which prevented the previous shift of blood flow away from the renal cortex. However, since no control infusion with saline was done, this may be an effect of intraarterial infusion per se, rather than an effect of mannitol or crossclamping. Furthermore, when mannitol was discontinued at declamping, cortical flow decreased to the initial clamp level rather than to the baseline level, further supporting the concept that the mannitol infusion caused these changes in flow distribution, independent of aortic clamping-declamping.

Berkowitz and Shetty [7] reported no change in flow to xenon component $C_{I}$ (cortex), but a decrease in the percentage of isotope distributed to $C_{1}$, with an increase in both flow and percentage distribution to $\mathrm{C}_{\mathrm{II}}$ (juxtamedullary and outer medulla) during aortic clamping. However, in the absence of operated controls (without clamp- ing) these data are consistent with decreased cortical flow over time, as described above. Although a bolus of saline was given initially, if sufficient fluids were not provided continuously during the experiment this could again explain the shift of blood flow away from the cortex. The finding [7] of increased renal vein renin activity differs from the present report and suggests that the preparation was different in some respects.

The overriding question is whether the xenon washout technique used in the above studies is a valid indicator of intrarenal blood flow distribution. The validity of xenon washout to determine the intrarenal distribution of blood flow rests on many assumptions which are beyond the scope of this paper. However, changes in the diffusion constant and kidney volume associated with changes in kidney water content and urine flow [19], axial streaming produced by intraarterial injection [4], and the difficulty in assigning washout curve components to specific anatomic areas under abnormal states $[10,12,13,29]$ have been described in detail as noted. Xenon component $C_{I}$ is a measure of total cortical flow, which corresponds to total flow per gram (Table 3) when measured by microspheres. Component II is thought to represent juxtamedullary and outer medullary regions. This would best correspond to Zone 4 by microsphere determination, since medullary blood flow is derived from inner cortical regions. While microsphere regions remain constant anatomically, this is not necessarily true with xenon washout. Xenon washout curves combine all anatomic areas with the same flow/ volume relationship into one exponential component. Thus, under varying experimental conditions, the anatomic areas assigned to these components might change. Only concomitant autoradiography allows any extrapolation from washout curves to definite anatomic areas. In fact, Carriere has shown by autoradiography that the $C_{I I}$ component of the washout curve cor- 
responds to outer medullary flow in control periods, but switches to a slow cortical component under various experimental conditions [12]. Slotkoff et al. were unable to correlate $\mathrm{C}_{\mathrm{II}}$ flow with any specific area of kidney cortex [37]. Studies by Shanser et al. attempted to correlate xenon determination of flow distribution with microsphere techniques and arteriograms in dogs during hemorrhagic hypotension [35]. Xenon curves suggested a loss of $C_{I}$, implying marked decrease in cortical flow. Yet microsphere analysis and arteriography showed uniform reduction of blood flow to all renal zones with no distribution away from the cortex. Mowat et al. demonstrated that intraarterial bolus injection of xenon produces a marked fall in renal blood flow measured by flowmeter [25]. In fact, flow measured by xenon washout correlated with the markedly reduced flow, not with normal flow. These authors concluded that this criticism precludes the use of Xenon washout for compartmental analysis. In light of this substantial criticism of the xenon washout technique, and in the absence of autoradiographic confirmation, the conclusion by Abbott and Berkowitz and their colleagues that renal blood flow distribution changed at aortic clamping is subject to question.

Several recent reports have utilized additional techniques to evaluate renal function during aortic cross-clamping. Renal oxidative enzymes were depressed with increased duration of cross-clamping [21]. However, this unfortunately was not correlated with standard physiologic data which would allow interpretation with respect to the similarity of this and other preparations. Reports of increased lysozymuria in patients undergoing aortic surgery suffers the same defect [32]. These increases, in fact, though representing renal tubular damage, may not exist in patients with adequate salinc replacement. At any rate, without knowledge of cardiac output, blood pressure, and other kidney function parameters, it is impossible to evaluate the general applicability of these findings. Although the use of mannitol has been advocated to prevent renal malfunction during aortic surgery $[2,5,14,30]$, no specific effect on kidney vasculature apart from its expansion of extracellular space has been proven. Other studies have demonstrated no beneficial effect of mannitol over general saline replacement in adequate amounts $[6,18]$. Although increased total renal blood flow occurs, no redistribution of flow exists [24]. A recent report of hypertonic mannitol in high doses ameliorating renal cell swelling in rats following total renal ischemia [14] is probably not applicable to infrarenal clamping and has not been evaluated in patients. In the presence of a contracted extracellular space with oliguria during cross-clamping of the aorta, mannitol may prevent sludging of cellular debris in renal tubules and decrease cellular swelling. However, without saline replacement, this effect will soon aggravate the underlying condition as osmotic diuresis occurs. Loop diuretics accomplish the same results and, in addition, have the theoretical advantage of increasing flow to the outer cortex [10, 24]. Nevertheless, avoiding these situations by adequate saline replacement is probably the simplest and safest method of avoiding renal damage during aortic cross-clamping.

\section{CONCLUSION}

In the presence of adequate extracellular fluid replacement, sufficient to prevent hypotension and to maintain normal renal blood flow, normal cardiac output, and normal urine output, no change in the distribution of intrarenal blood flow could be demonstrated during clamping or declamping the infrarenal aorta. Recommendations by other investigators that mannitol be used to prevent renal cortical ischemia are not well supported by experimental observations. Adequate intraoperative saline and blood replacement, as indicated by monitoring cardiac output, blood 
pressure, and urine output, appear adequate to prevent impairment of renal function. No adverse renal effect of aortic crossclamping could be demonstrated in this study.

\section{ACKNOWLEDGMENTS}

The authors gratefully acknowledge the assistance of Mr. John Jones, Phoenix Memorial Laboratory, for isotope analysis; Mr. James Lepkowski and Dr. George Williams, School of Public Health, for statistical analysis; and Dr. Theodore Beals, Veterans Administration Hospital, for histological examination.

\section{REFERENCES}

1. Abbott, W. M., Cooper, J. D., and Austen, W. G. The effect of aortic clamping and declamping on renal blood flow distribution. $J$. Surg. Res. 14: 385, 1972.

2. Abbott, W. M., and Austen, W. G. The reversal of renal cortical ischemia during aortic occlusion by mannitol. J. Surg. Res. 16: 482, 1974.

3. Arruda, J. A. L., Boonjaren, S., Westenfelder, C., and Kurtzman, N. A. Measurement of renal blood flow with radioactive microspheres. Proc. Soc. Exp. Biol. Med. 146: 263, 1974.

4. Aukland, K. Effect of adrenalin, noradrenalin, angiotensin, and renal nerve stimulation on intrarenal distribution of blood flow in dogs. Acta. Physiol. Scand. 72: 498, 1968.

5. Barry, K. G., Cohen, A., Knochel, J. P., Whelan, T. J., Beisel, W. R., Vargas, C. A., and LeBlanc, P. C. Mannitol infusion. II. The prevention of acute functional renal failure during resection of an aneurysm of the abdominal aorta. N. Engl. J. Med. 264: 967, 1961.

6. Beall, A. C., Hall, C. W., Morris, G. C., and DeBakey, M. E. Mannitol induced osmotic diuresis during renal artery occlusion. Ann. Surg. 161: $46,1965$.

7. Berkowitz, H. D., and Shetty, S. Renin release and renal cortical ischemia following aortic cross clamping. Arch. Surg. 109: 612, 1974.

8. Berkowitz, H. D., Galvin, C. G., and Miller, L. D. The control of renal cortical perfusion by the renin angiotensin system. Ann. Surg. 179: 238, 1974.

9. Berry, W. M., Morris, G. C., and DeBakey, M. E. Alterations in renal hemodynamics during surgical resection of abdominal aortic aneurysms. Clin. Res. 1: 163, 1959.

10. Birtch, A. G., Zakheim, R. M., Jones, L. G., and Barger, A. C. Redistribution of renal blood flow produced by furosemide and ethacrynic acid. Circ. Res. 21: 869, 1967.

11. Buckberg, G. D., Luck, J. C., Payne, D. B.,
Hoffman, J. I. E., Archie, J. P., and Fixler, D. E. Some sources of error in measuring regional blood flow with radioactive microspheres. J. Appl. Physiol. 31: 598, 1971.

12. Carriere, S. Effect of norepinephrine, isoproterenol, and adrenergic blockers upon the intrarenal distribution of blood flow. Canad. J. Physiol. Pharmacol. 47: 199, 1969.

13. Carriere, S., Thorburn, G. D., O'Morchoe, C. C. C., and Barger, A. C. Intrarenal distribution of blood flow in dogs during hemorrhagic hypotension. Circ. Res. 19: 167, 1966.

14. Flores, J., DiBona, D. R., Beck, C. H., and Leaf, $A$. The role of cell swelling in ischemic renal damage and the protective effect of hypertonic solute. J. Clin. Invest. 51: 118, 1972.

15. Foster, J. H., Adkins, R. B., Chamberlain, N. O., Symbas, P. N., and Harris, A. P. The renal effects of lower abdominal aortic cross-clamping. Report of negative results in dogs and monkeys. JAMA 183: 451, 1963.

16. Fry, W. J., Keitzer, W. F., Kraft, R. O., and DeWeese, M. S. Prevention of hypotension due to aortic release. Surg. Gynecol. Obstet. 116: $301,1963$.

17. Gagnon, J. A., Bolt, D. A., Clarke, R. W., and Geever, E. F. The effects of lower aortic occlusion on renal function in the dog. Surgery 47: $240,1960$.

18. Hutchin, P., Terzi, R. G., and Hollandsworth, L. C. Renal response to intraoperative fluid administration. Surg. Gynecol. Obstet. 129: 794, 1969.

19. Katz, M. A., Blantz, R. C., Rector, F. C., and Seldin, D. W. Measurement of intrarenal blood flow. I. Analysis of microsphere method. Amer. J. Physiol. 220: 1903, 1971.

20. Kountz, S. L., Tuttle, K. L., Cohn, L. H., Eschelman, B. A., and Cohn, R. Factors responsible for acute tubular necrosis following lower aortic surgery. JAMA 183: 447, 1963.

21. Lempert, N., Pakdaman, P., Stein, A., Boba, A., and Powers, S. R., Jr. Further studies on enzymatic histochemical alterations in the renal tubular epithelium of dogs. II. The effect of aortic crossclamping below the renal arteris. III. The effect of pretreatment with $10 \%$ mannitol in peripheral trauma. Ann. Surg. 160: 115, 1964.

22. McGonigle, D. J., Seiple, R. S., and Wylie, E. J. Renal effects of temporary extensive infrarenal arterial occlusion: An experimental study. Surgery 49: 235, 1961.

23. McNay, J. L., and Abe. Y. Pressure-dependent heterogeneity of renal cortical blood flow in dogs. Circ. Res. 27: 571, 1970.

24. McNay, J. L., and Abe, Y. Redistribution of cortical blood flow during renal vasodilatation in dogs. Circ. Res. 27: 1023, 1970. 
25. Mowat, P., Lupu, A. N., and Maxwell, M. H. Limitations of ${ }^{133} \mathrm{Xe}$ washout technique in estimation of renal blood flow. Amer. J. Physiol. 223: $682,1972$.

26. Mowlen, A., McClintock, J. T., and Campbell, G. S. The effect on renal function of occlusion of the aorta inferior to the renal vessels. Surg. Gynecol. Obstet. 111: 423, 1960.

27. Nanson, E. M., and Noble, J. G. The effect on the kidneys of cross-clamping the abdominal aorta distal to the renal arteries. Surgery 46: 288, 1959.

28. Perry, M. O. The hemodynamics of temporary abdominal aortic occlusion. Ann. Surg. 168: 193, 1968.

29. Pomeranz, B. H., Birtch, A. G., and Barger, A. C. Neural control of intrarenal blood flow. Amer. J. Physiol. 215: 1067, 1968.

30. Porter, J. M., McGregor, F., Acinapura, A. J., and Silver, D. Renal function following abdominal aortic aneurysmectomy. Surg. Gynceol. Obstet. 123: $819,1966$.

31. Powers, S. R., Boba, A., and Stein, A. The mechanism and prevention of distal tubular necrosis following aneurysmectomy. Surgery 42: 156, 1957.

32. Powis, S. J. A. Renal function following aortic surgery. J. Cardiovasc. Surg. (Torino) 16: 565, 1975.

33. Rector, J. B., Stein, J. H., Bay, W. H., Osgood, R. W., and Ferris, T. F. Effect of hemorrhage and vasopressor agents on distribution of renal blood flow. Amer. J. Physiol. 222: 1125, 1972.

34. Rudolph, A. M., and Heymann, M. A. The circulation of the fetus in utero. Methods for studying distribution of blood flow, cardiac output and organ blood flow. Circ. Res. 21: 163, 1967.

35. Shanser, J. D., Korobkin, M., Seidlitz, L., Carlson, E. L., and Shames, D. M. Hazards in interpreation of xenon washout. Studies of the canine kidney. Radiology 11: 461, 1974.

36. Slater, P. V. and Hayes, M. A. Renal function during infrarenal cross-clamping of the aorta, Rocky Mt. Med. J. 66: 50, (July) 1969.

37. Slotkoff, L. M., Logan, A., Jose, P., D'Avella, J., and Eisner, G. M. Microsphere measurement of intrarenal circulation of the dog. Circ. Res. 28: $158,1971$.

38. Stein, J. H., Ferris, T. F., Huprich, J. E., Smith, T. C., and Osgood, R. W. Effect of vasodilatation on the distribution of cortical blood flow in the kidney of the $\operatorname{dog} . J$. Clin. Invest. 50: $1429,1971$.

39. Stein, J. H., Boonjaren, S., Wilson, C. B., and Ferris, T. F. Alterations in intrarenal blood flow distribution. Methods of measurement and relationship to sodium balance. Circ. Res. 32-33 (Suppl 1): I-61, 1973.

40. Stein, M., James, P. M. Kelly, J., Brown, D., Shircliffe, A. C., and Patterson, W. E. Renal protection during aortic cross-clamping. Amer. Surg. 38: 681, 1972.

41. Thompson, J. E., Vollman, R. W., Austin, D. J., and Kartehner, M. M. Prevention of hypotensive and renal complications of aortic surgery using balanced salt solution: Thirteen-year experience with 670 cases. Ann. Surg. 167: 767, 1968. 